Taxonomía y sistemática

\title{
Descripción de la genitalia de Coptocycla (Psalidonota) leprosa (Chrysomelidae: Cassidinae: Cassidini)
}

\author{
Description of the genitalia of Coptocycla (Psalidonota) leprosa (Chrysomelidae: \\ Cassidinae: Cassidini)
}

Sara López-Pérez

Departamento de Zoología, Instituto de Biología, Universidad Nacional Autónoma de México, Apartado postal 70-153, 04510 Ciudad de México, México

Recibido el 11 de enero de 2016; aceptado el 5 de abril de 2016

Disponible en Internet el 6 de agosto de 2016

\section{Resumen}

Se describe la genitalia de Coptocycla (Psalidonota) leprosa (Boheman, 1855). El edeago presenta la parte apical ensanchada seguida de una quilla en vista dorsal, carácter que difiere del esquema general registrado previamente para los genitales masculinos de la subfamilia Cassidinae. Los genitales femeninos siguen el patrón definido para la mayoría de los casidinos.

Derechos Reservados @ 2016 Universidad Nacional Autónoma de México, Instituto de Biología. Este es un artículo de acceso abierto distribuido bajo los términos de la Licencia Creative Commons CC BY-NC-ND 4.0.

Palabras clave: Edeago; Saco interno; Endofalo; Cápsula espermática; Velum

\begin{abstract}
The genitalia of Coptocycla (Psalidonota) leprosa (Boheman, 1855) are described. This species differs from the pattern of male genitalia for Cassidinae. The aedeagus of $C$. leprosa is characterized for show the apical portion wide, and a keel in the apical portion in dorsal view. The female genitalia do not differ from the pattern proposed for Cassidinae. This study confirms the need for more comprehensive studies of the genitalia of beetles and standardizes the terminology used.

All Rights Reserved (C) 2016 Universidad Nacional Autónoma de México, Instituto de Biología. This is an open access item distributed under the Creative Commons CC License BY-NC-ND 4.0.
\end{abstract}

Keywords: Aedeagus; Internal sac; Endophallus; Spermatheca; Velum

\section{Introducción}

Los genitales de los insectos presentan variación morfológica y es un conjunto de estructuras que diverge rápidamente (Eberhard, 1985; Zunino, 2012). Desde principios del siglo Xx, la morfología de los genitales ha sido utilizada en los insectos como uno de los caracteres principales para identificar especies. En la mayoría de los trabajos taxonómicos en insectos, los genitales masculinos son la estructura más utilizada. Coleoptera no

Correo electrónico: slopez.p@hotmail.com

La revisión por pares es responsabilidad de la Universidad Nacional Autónoma de México. es la excepción, en este grupo los genitales son de gran utilidad para diferenciar especies, incluso especies crípticas con hábitats superpuestos (Manjarrés-Hernández y Molano-Rendón, 2013).

El primer trabajo que aborda las estructuras reproductoras de Coleoptera es el de Sharp y Muir (1912), quienes compararon la morfología de los genitales masculinos y discutieron las relaciones filogenéticas de las familias. Para Chrysomelidae existen varios trabajos: Zia (1936) estudió la morfología de los genitales de diferentes subfamilias, Powell (1941) describió la morfología de 74 especies, Suzuki (1988) realizó un estudio comparativo de la morfología de los genitales y discutió las relaciones filogenéticas entre subfamilias, el mismo autor en 1989 (Suzuki, 1989) estudió la correlación del sistema reproductivo de la familia, Mann y Crowson (1996) contribuyeron con el estudio del saco 
interno de diversos crisomélidos y Verma (1996), a partir de una revisión bibliográfica sobre los genitales masculinos, analizó las relaciones filogenéticas entre las subfamilias.

Recientemente, dentro de la subfamilia Cassidinae se ha observado que la variación en la morfología de la genitalia ofrece caracteres con valor diagnóstico y filogenético (Borowiec y Opalinska, 2007; Borowiec y Świętojańska, 2001; Chaboo, 2001; Rodríguez, 1994a, b; Shin y Chaboo, 2012; Shin, Chaboo y Clark, 2012; Simões, 2012; Świętojańska, 2001). En el género Chelymorpha Chevrolat, algunas especies presentan un alto grado de polimorfismo y la cápsula espermática es determinante para distinguir entre las especies, ya que es una estructura con una morfología constante dentro de cada especie (Borowiec y Skuza, 2004). Sin embargo, a pesar del reciente aumento de estudios de los genitales en Cassidinae, aún existe gran carencia de información sobre las estructuras internas. Por ejemplo, para la tribu Cassidini son escasos los trabajos que abordan los genitales (Sekerka, 2005; Suenaga, 2013), siendo paradójicamente una de las tribus más diversas dentro de la subfamilia.

El presente estudio tiene como objetivo contribuir al conocimiento de los genitales en especies de Cassidinae, describiendo los genitales masculinos y femeninos de Coptocycla (Psalidonota) leprosa (Boheman, 1855) (fig. 1.1, 1.2).

\section{Materiales y métodos}

Se examinaron 70 ejemplares pertenecientes a la Colección Nacional de Insectos del Instituto de Biología, UNAM. Para la extracción de la genitalia, los ejemplares se hidrataron en agua caliente por aproximadamente $1 \mathrm{~h}$. Se separó el abdomen y se colocó en $\mathrm{KOH}$ al $10 \%$ en agua tibia durante 15 min. Después de la maceración, el abdomen se lavó con agua corriente. Posteriormente se realizó una incisión en la pleura, lo cual permitió abrirlo y extraer los genitales de machos y hembras que se colocaron en microviales con glicerina para su preservación.
Para nombrar las estructuras se siguió la terminología de Chaboo (2007), Suzuki (1988) y Verma y Kumar (1972). Se usó un microscopio estereoscopio Zeiss Discovery V8. Las fotografías digitales fueron tomadas en un estereoscopio Axio Zoom V16 y las micrografías se tomaron con el microscopio electrónico de barrido Hitachi S-2460 N (15KV).

\section{Descripción}

Genitales masculinos. Edeago (lóbulo medio) tubular, curvo. Longitud $(\mathrm{N}=34)$ : $2.4 \mathrm{~mm}$ en promedio $(\sigma=0.217)$ (fig. 2.3 ); proporción longitud total del macho-longitud del edeago 100:20. Parte apical ensanchada lateralmente, de 1.5 a 2 veces el ancho de la base, inclinada; paredes laterales con pequeñas puntuaciones dispersas; proceso apical pequeño, truncado (fig. 2.4). Ostium (orificio medio) en forma de campana; a través del ostium se puede observar la parte apical del saco interno (= endofalo, Snodgrass, 1935), el cual en vista frontal es voluminoso y membranoso, con elevaciones ventrales (EISV), laterales (EISL) y dorsales (EISD), sin espinas ni microestructuras (fig. 2.4). Parte media delgada lateralmente, dorsalmente con una quilla que termina en la apertura del ostium, serie de estrías paralelas a los costados de la quilla que se unen donde inicia la quilla (fig. 2.5). Spiculum gastrale (esternito 9) esclerosado, en forma de horquilla (fig. 2.6). Parte basal levemente constreñida en vista dorsoventral. Tegmen en forma de «Y» rodeando la base del edeago (fig. 2.7); manubrio poco más corto que los brazos, borde presente en la parte ventral (fig. 2.8). Ducto eyaculador largo, liso, aproximadamente 10 veces el largo del edeago.

Genitales femeninos. Cápsula espermática fuertemente esclerosada, en forma de hoz (fig. 3.9). Longitud ( $\mathrm{N}=36)$ : $0.6 \mathrm{~mm}$ en promedio $(\sigma=0.040)$; proporción longitud total de la hembralongitud de la cápsula espermática 100:5. Espacio entre el ápice y la luz menos de la mitad de la longitud de la cápsula espermática. Receptáculo de mayor diámetro que la parte distal, menos

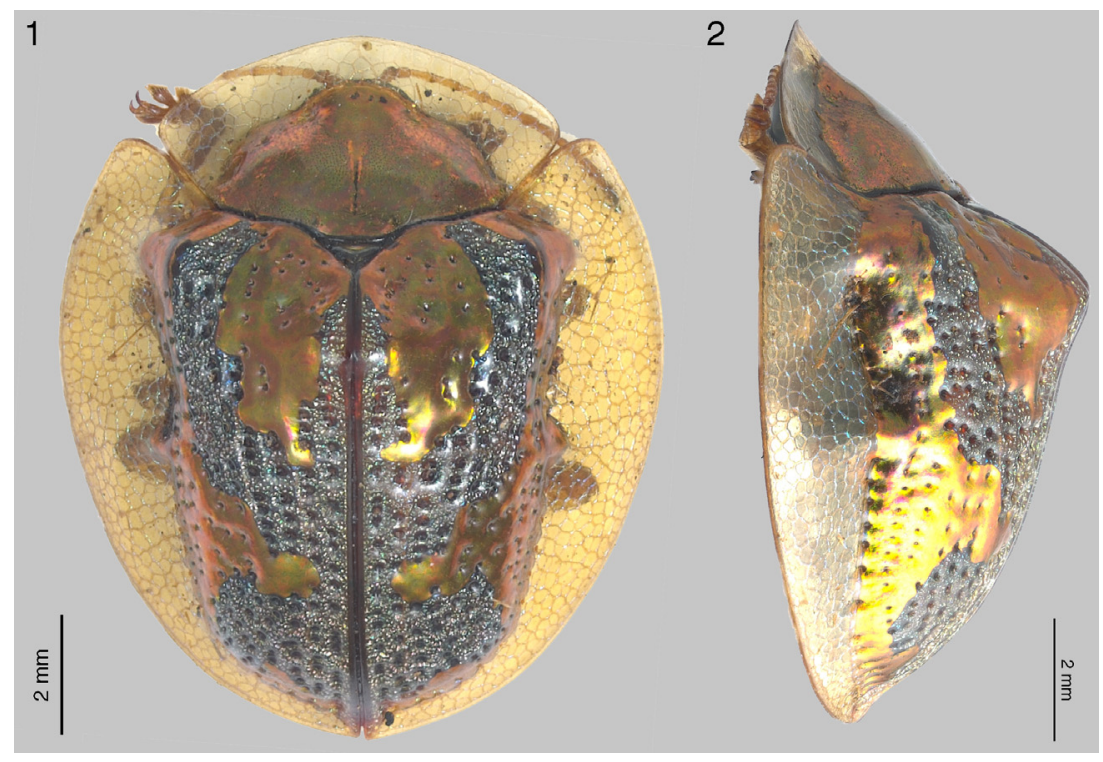

Figura 1. Coptocycla (Psalidonota) leprosa, $\sigma^{7}$ vista dorsal y lateral. 


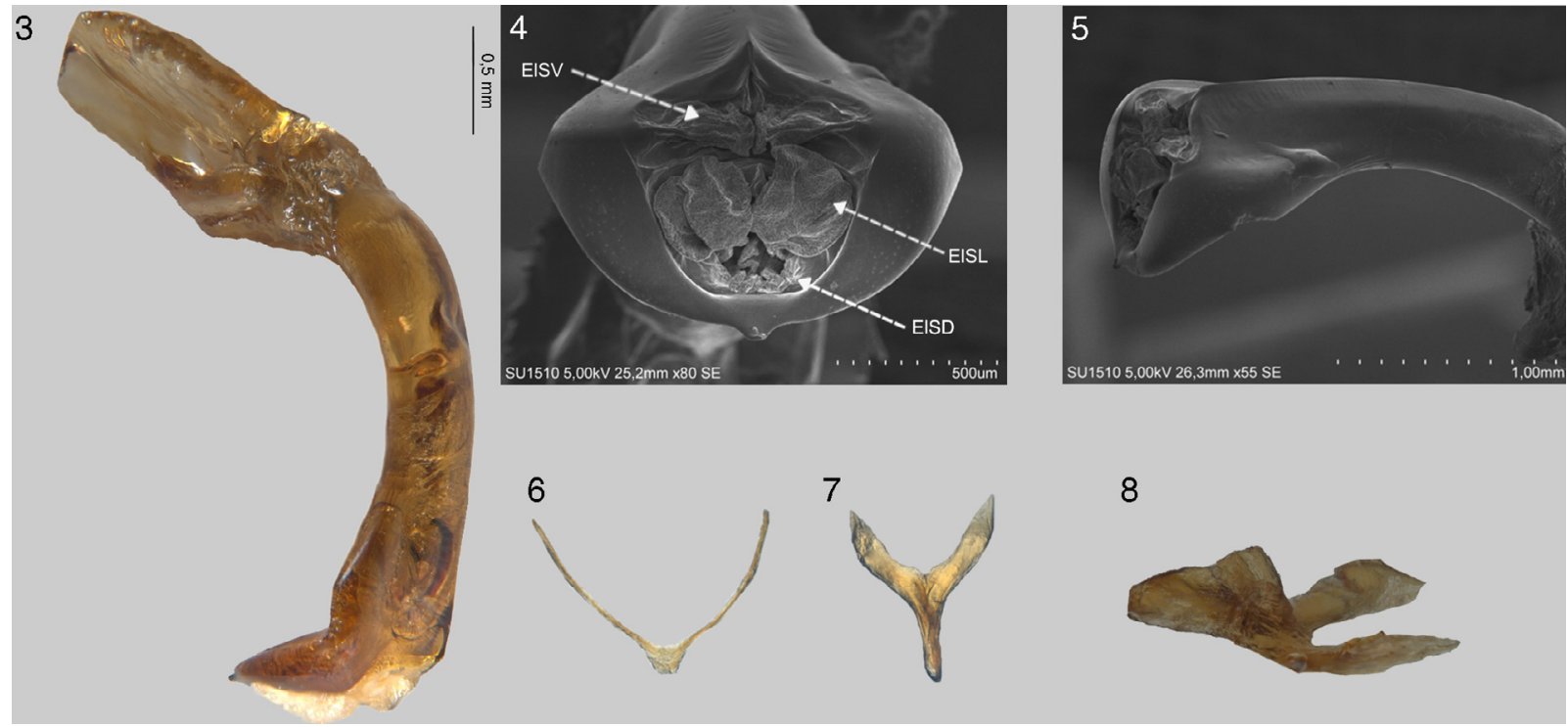

Figura 2. Genitales masculinos de Coptocycla (Psalidonota) leprosa: 3, edeago en vista lateral; 4, parte apical del edeago; 5 , edeago en vista ventrolateral; 6 , spiculum gastrale; 7, tegmen; 8, tegmen en vista ventrolateral.

de la mitad que la longitud de la cápsula espermática; ápice agudo con velum; ducto espermático liso; glándula espermática presente, próxima al ducto espermático. Ducto y glándula espermática insertados en parte basal de la cápsula espermática, en una especie de vesícula (fig. 3.10) (= ramus; Santos y RosadoNeto, 2010). Esternito VIII con sedas esparcidas y largas; ápice casi recto, con pubescencia cerrada (fig. 3.11).

\section{Discusión}

La conformación de los genitales masculinos es distinta en cada una de las familias de Chrysomeloidea (Reid, 1995). En Chrysomelidae se compone básicamente del edeago, tegmen, spiculum gastrale y conducto eyaculatorio; los parámeros (lóbulos laterales) están ausentes (Düngelhoef y Schmitt, 2010). Suzuki (1988) menciona que la mayoría de los edeagos de la subfamilia Cassidinae son largos y delgados o en ocasiones cortos y gruesos. Este patrón que se ha observado en la mayoría de las tribus de Cassidinae, incluyendo Cassidini, tribu a la cual pertenece C. leprosa, ha sido constante (Borowiec, 2007; Sassi y Borowiec, 2006; Sekerka, 2005; Suenaga, 2013; obs. pers.). Sin embargo, C. leprosa rompe el esquema observado en la subfamilia, al presentar la parte apical notablemente ensanchada lateralmente, este carácter se documenta por primera vez para Cassidinae y también se encuentra en otras especies de Coptocycla, como C. dorsoplagiata Champion, 1894 y C. texana

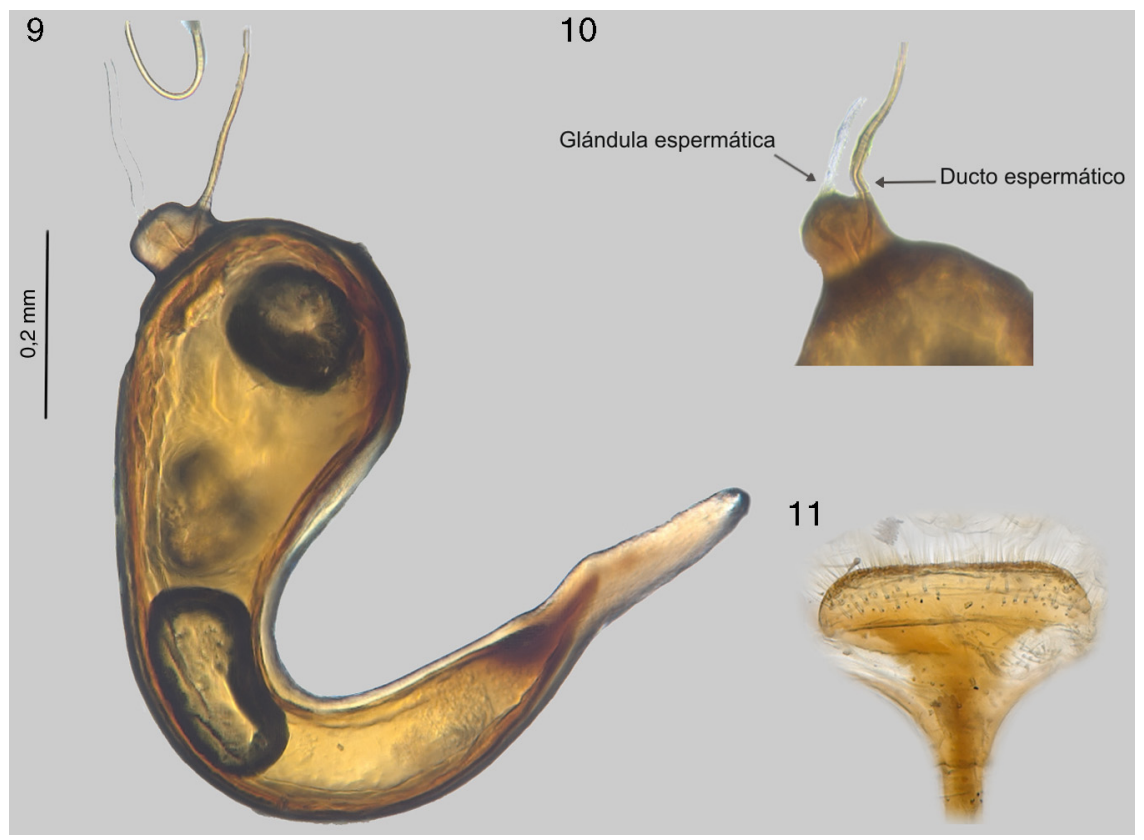

Figura 3. Genitales femeninos de Coptocycla (Psalidonota) leprosa: 9, cápsula espermática; 10, ramus; 11, esternito VIII. 
(Schaeffer, 1933). Otros caracteres propios de C. leprosa son las puntuaciones dispersas en las paredes laterales, la quilla y estrías en la parte media dorsal.

La conformación del saco interno en Chrysomelidae es variada. En Lilioceris lilii (Scopoli, 1763) y Oulema duftschmidi (Redtenbacher, 1874) (Criocerinae) se observan microestructuras en la superficie del saco. Las microestructuras parecieran tener la función de impedir la separación cuando la hembra intenta detener la cópula (Düngelhoef y Schmitt, 2006). Asimismo, otras especies de Lema Fabricius, 1798 (Criocerinae) y de la subfamilia Donaciinae presentan un saco interno con espinas, que probablemente cumplan con la misma función (Matsumura y Suzuki, 2008). En Cassidinae también se pueden encontrar espinas y/o pubescencia, sin embargo, C. leprosa no presentó ninguna ornamentación. Hasta el momento no se ha reportado alguna estrategia para C. leprosa que le permita retener a la hembra. Probablemente esta especie utiliza las sedas presentes en los tarsómeros, ya que le permiten adherirse a los élitros de las hembras al momento de la cópula. Una estrategia reproductiva que le concede ventaja a $C$. leprosa es el ducto eyaculatorio largo; se ha observado que este asegura una transferencia de esperma directa, ya sea dentro o cerca del receptáculo de la cápsula espermática (Düngelhoef y Schmitt, 2006; Rodríguez, Windsor y Eberhard, 2004).

En las hembras la forma de la cápsula espermática puede ser distinta entre especies. La forma de hoz es una de las más comunes dentro de la familia Cassidinae, la cual presenta C. leprosa. En otras especies de Trichaspis Spaeth, 1911, Chiridopsis Spaeth, 1922, Glyphocasis Spaeth, 1914 e Hybosinota Spaeth, 1909, la cápsula espermática no está fuertemente esclerosada o el receptáculo es del mismo diámetro que la parte distal (fig. 4.12-15). Además de la forma, el número de receptáculos varía dentro de la subfamilia Cassidinae. En Eugenysa columbiana (Boheman, 1850) se presentan 3 receptáculos, un número constante dentro de la tribu Eugenysini (Chaboo, 2002), a diferencia de $C$. leprosa en donde solo hay uno. Otra estructura que varía es el ducto espermático; en algunas especies puede tener forma de espiral, plegado o bien puede ser completamente liso como en $C$. leprosa. El ducto espermático y la glándula en ocasiones se encuentran en un mismo punto de comunicación en el receptáculo. Sin embargo, en otras especies pueden encontrarse en partes distales, como p. ej. Aspidimorpha miliaris (Fabricius, 1775).

La variación en la morfología de los genitales ofrece información útil para estudios taxonómicos. El presente trabajo no solo aporta la descripción morfológica de los genitales masculinos y femeninos, sino también nuevos caracteres que difieren del esquema general del edeago para Cassidinae e incluso de Chrysomelidae, ya que la parte apical ensanchada en el edeago de C. leprosa no se había referido hasta el momento, lo mismo que la quilla presente en la parte apical en vista dorsal. Además de su uso diagnóstico, los genitales tanto de machos como de hembras son útiles en análisis filogenéticos. La homogeneización de la terminología y el aumento de descripciones morfológicas permitirá contar con una base amplia de caracteres útiles en el estudio de las relaciones filogenéticas de especies, géneros y subfamilias.

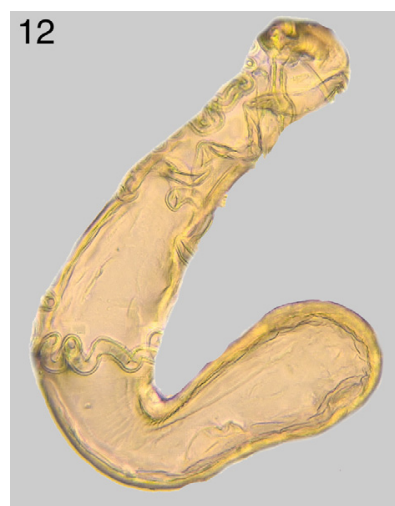

13

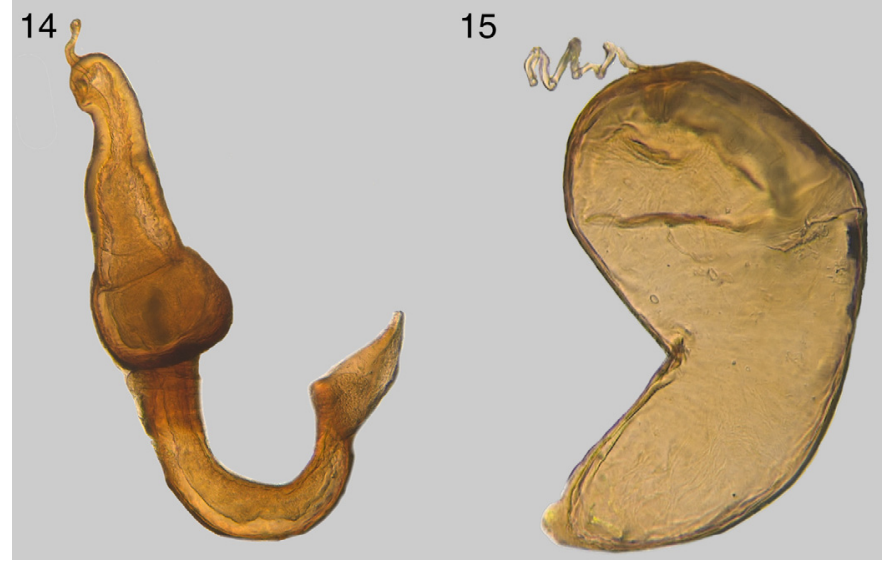

Figura 4. Cápsulas espermáticas de distintas hembras de Cassidinae: 12, Trichaspis pilasula (Boh.); 13, Chiridopsis aubei (Boh.); 14, Glyphocasis lepida (Spaeth); 15, Hybosinota nodulosa (Boh.)

\section{Agradecimientos}

A Santiago Zaragoza Caballero y Alfonso N. García Aldrete por sus valiosos comentarios. A Susana Guzmán por la asistencia técnica en la toma de fotografías. A M. Berenit Mendoza por la toma de micrografías de microscopia electrónica. A 2 revisores anónimos por sus atinados comentarios. Al Programa de Posgrado en Ciencias Biológicas de la Universidad Nacional Autónoma de México.

\section{Referencias}

Borowiec, L. (2007). A new species of Microctenochira Spaeth from Belize (Coleoptera: Chrysomelidae: Cassidinae: Cassidini). Genus, 18, 487-492.

Borowiec, L. y Opalinska, S. (2007). The structure of spermathecae of selected genera of Stolaini and Eugenysini (Coleoptera: Chrysomelidae: Cassidinae) and its taxonomic significance. Annales Zoologici, 57, 463-479.

Borowiec, L. y Skuza, M. (2004). The structure of spermatheca in the genus Chelymorpha Chevrolat, 1837 (Coleoptera: Chrysomelidae: Cassidinae) and its taxonomic significance. Annales Zoologici, 54, 439-451.

Borowiec, L. y Świętojańska, J. (2001). Revision of Cassida litigiosa group from southern Africa (Coleoptera: Chrysomelidae: Cassidinae). Annales Zoologici, 51, 153-184.

Chaboo, C. S. (2001). Revision and phylogenetic analysis of Acromis Chevrolat (Coleoptera: Chrysomelidae: Cassidinae: Eugenysini). The Coleopterists Bulletin, 55, 50-67.

Chaboo, C. S. (2002). First report of immatures, genitalia and maternal care in Eugenysa columbiana (Boheman) (Coleoptera: Chrysomelidae: Cassidinae: Eugenysini). The Coleopterists Bulletin, 56, 50-67. 
Chaboo, C. S. (2007). Biology and phylogeny of the Cassidinae Gyllenhal sensu lato (tortoise and leaf-mining beetles) (Coleoptera: Chrysomelidae). Bulletin of the American Museum of Natural History, 305, 1-250.

Düngelhoef, S. y Schmitt, M. (2006). Functional morphology of copulation in Chrysomelidae-Criocerinae and Bruchidae (Insecta: Coleoptera). Bonner Zoologische Beiträge, 54, 201-208.

Düngelhoef, S. y Schmitt, M. (2010). Genital feelers: the putative role of parameres and aedeagal sensilla in Coleoptera Phytophaga (Insecta). Genetica, $138,45-57$.

Eberhard, W. G. (1985). Sexual selection and animal genitalia. Cambridge: Harvard University Press.

Manjarrés-Hernández, E. H. y Molano-Rendón, F. (2013). Piezas genitales masculinas como carácter taxonómico en escarabajos coprófagos (Coleoptera: Scarabaeidae). Revista Ciencia en Desarrollo, 4, 39-47.

Mann, J. S. y Crowson, R. A. (1996). Internal sac structure and phylogeny of Chrysomelidae. En P. H. Jolivet y M. L. Cox (Eds.), Chrysomelidae biology, Vol. 1. Classification, phylogeny and genetics (pp. 291-316). Amsterdam: Academic Publishing.

Matsumura, Y. y Suzuki, K. (2008). Comparative morphology of internal reproductive systems in leaf beetles of the Donaciinae and Criocerinae (Coleoptera: Chrysomelidae) and its implication for the phylogeny. Zootaxa, $1845,1-32$.

Powell, E. F. (1941). Relationships within the family Chrysomelidae (Coleoptera) as indicated by the male genitalia of certain species. American Midland Naturalist, 25, 148-195.

Reid, C. A. M. (1995). A cladistic analysis of subfamilial relationships in the Chrysomelidae sensu lato (Chrysomeloidea). Biology, phylogeny and classification of Coleoptera: papers celebrating the 80th birthday of Roy A. Crowson, 2, 559-631.

Rodríguez, V. (1994a). Sexual behavior in Omaspides convexicollis Spaeth and $O$. bistriata Boheman, with notes on maternal care of eggs and young. Coleopterists Bulletin, 48, 140-144.

Rodríguez, V. (1994b). Function of the spermathecal muscle in Chelymorpha alternans Boheman (Coleoptera: Chrysomelidae: Cassidinae). Physiological Ecology, 19, 198-202.

Rodríguez, V., Windsor, D. M. y Eberhard, W. G. (2004). Tortoise beetle genitalia and demonstrations of a sexually selected advantage for flagellum length in Chelymorpha alternans (Coleoptera, Cassidini, Stolaini). En P. Jolivet, J. A. Santiago-Blay, y M. Schmitt (Eds.), New developments in the biology of Chrysomelidae (pp. 739-748). La Haya: SPB Academic Publishing bv.

Santos, G. B. D. y Rosado-Neto, G. H. (2010). Morphological aspects of the genitalia of seven species of Heilus Kuschel (Coleoptera, Curculionidae). Revista Brasileira de Entomologia, 54, 157-164.
Sassi, D. y Borowiec, L. (2006). Cassida inopinata, a new species from Italy and Balkan region (Coleoptera: Chrysomelidae: Cassidinae). Genus, 17 $545-560$.

Sekerka, L. (2005). Cassida olympica, a new species from Greece (Coleoptera: Chrysomelidae: Cassidinae). Genus, 16, 285-289.

Sharp, D. y Muir, F. A. G. (1912). The comparative anatomy of the male genital tube in Coleoptera. Transactions of the Entomological Society of London, 3 , 477-642.

Shin, C. y Chaboo, C. S. (2012). A revision and phylogenetic analysis of Stoiba Spaeth 1909 (Coleoptera, Chrysomelidae). ZooKeys, 224, 1-53.

Shin, C., Chaboo, C. S. y Clark, S. M. (2012). Revision of the endemic Hispaniolan genus Asteriza Chevrolat, 1836, with description of two new species (Coleoptera: Chrysomelidae: Cassidinae: Ischyrosonychini). Zootaxa, 3227, 4-53.

Simões, M. V. (2012). Male and female reproductive systems of Stolas conspersa (Germar) (Coleoptera, Chrysomelidae, Cassidinae). Revista Brasileira de Entomologia, 56, 19-22.

Snodgrass, R. E. (1935). Principles of insect morphology. New York: McGrawHill.

Suenaga, H. (2013). Notes on Cassida ferruginea and Cassida mongolica in Japan, with descriptions of their reproductive systems (Coleoptera: Chrysomelidae: Cassidinae). Genus, 24, 325-333.

Suzuki, K. (1988). Comparative morphology of the internal reproductive system of the Chrysomelidae (Coleoptera). En P. Jolivet, E. Petitpierre, y T. H. Hsiao (Eds.), Biology of the Chrysomelidae (pp. 317-355). Dordrecht: Kluwer Academic Publishers.

Suzuki, K. (1989). Character correlation in the internal reproductive system of the family Chrysomelidae (Coleoptera). En D. G. Furth y T. N. Seeno (Eds.), Second International Symposium of the Chrysomelidae (pp. 511518). Entomography, 6.

Świętojańska, J. (2001). A revision of the tribe Aspidimorphini of the Oriental Region (Coleoptera: Chrysomelidae: Cassidinae). Genus, 11, 1-318.

Verma, K. K. (1996). Inter-subfamily relations among Chrysomelidae (Coleoptera) as suggested by organization of the male genital system. En P. H. A Jolivet y M. L. Cox (Eds.), Chrysomelidae Biology, Vol. 1. The classification, phylogeny and genetics (pp. 317-351). Amsterdam: Academic Publishing.

Verma, K. K. y Kumar, D. (1972). The aedeagus, its musculature, and 'retournement' in Aspidomorpha miliaris F. (Coleoptera, Phytophaga, Chrysomelidae). Journal of Natural History, 6, 699-719.

Zia, Y. (1936). Comparative studies of the male genital tube in Coleoptera Phytophaga. Sinensia, 7, 319-352.

Zunino, M. (2012). Cuarenta años de anatomía de las piezas genitales en la taxonomía de los escarabajos (Coleoptera: Scarabaeoidea): el estado del arte. Dugesiana, 18, 197-206. 